\title{
Oxidative stress mediated arterial dysfunction in patients with obstructive sleep apnoea and the effect of continuous positive airway pressure treatment
}

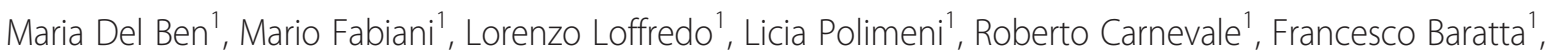 \\ Marco Brunori ${ }^{1}$, Fabiana Albanese ${ }^{1}$, Teresa Augelletti ${ }^{1}$, Francesco Violi ${ }^{1}$ and Francesco Angelico ${ }^{1,2^{*}}$
}

\begin{abstract}
Background: Several studies suggest an increase of oxidative stress and a reduction of endothelial function in obstructive sleep apnoea syndrome (OSAS). We assessed the association between OSAS, endothelial dysfunction and oxidative stress. Further aim was to evaluate the effect of nasal continuous positive airway pressure (nCPAP) on oxidative stress and arterial dysfunction.

Methods: We studied 138 consecutive patients with heavy snoring and possible OSAS. Patients underwent unattended overnight home polysomnography. Ten patients with severe OSAS were revaluated after 6 months of nCPAP therapy. To assess oxidative stress in vivo, we measured urinary 8-iso-PGF2a and serum levels of soluble NOX2-derived peptide (sNOX2-dp). Serum levels of nitrite/nitrate (NOx) were also determined. Flow-mediated brachial artery dilation (FMD) was measured to asses endothelial function.

Results: Patients with severe OSAS had higher urinary 8-iso-PGF2a $(p<0.001)$ and serum NOX2 and lower NOx. A negative association was observed between FMD and OSA severity. Apnea/hypopnea index was significantly correlated with the indices of central obesity and with urinary 8-isoprostanes $(r=0.298, p<0.001)$. The metabolic syndrome $(t=-4.63, p<0.001)$ and urinary 8 -isoprostanes $(t=-2.02, p<0.05)$ were the only independent predictors of FMD. After 6-months nCPAP treatment, a significant decrease of serum NOX2, $(p<0.005)$ and urinary 8-iso-PGF2a $(p<0.01)$ was observed, while serum NOx showed only a minor increase. A statistically significant increase of FMD was observed (from 3.6\% to $7.0 \%$ ).
\end{abstract}

Conclusions: The results of our study indicate that patients with OSAS and cardiometabolic comorbidities have increased oxidative stress and arterial dysfunction that are partially reversed by nCPAP treatment.

\section{Background}

Obstructive sleep apnoea syndrome (OSAS) is a common nocturnal disorder characterized by the presence of repetitive apnoea and hypopnoea during sleep, daytime sleepiness and cardiopulmonary dysfunction. Patients with OSAS experience recurrent episodes of cessation of breathing which expose the cardiovascular system

\footnotetext{
* Correspondence: francesco.angelico@uniroma1.it

'Department of Internal Medicine and Medical Specialities, Sapienza University, Rome, Italy

${ }^{2}$ Department of Public Health and Infectious Diseases, Division of Internal Medicine C, Policlinico Umberto 1, Viale del Policlinico 155, 00161 Rome, Italy
}

to cycles of hypoxia, exaggerated negative intrathoracic pressure and arousals [1].

The majority of OSAS patients show the cluster of metabolic and non-metabolic cardiovascular risk factors of the metabolic syndrome and it has also suggested that OSA may be a manifestation of metabolic syndrome (MS) [2-4].

Several studies have provided evidence supporting an increase of oxidative stress in OSAS [5-10]. Oxidative stress is characterized by an imbalance between oxidant and antioxidant mechanisms, in which many different enzymatic and non-enzymatic antioxidants take place. Recently, it has been postulated that intermittent hypoxia

\section{Biomed Central}


can induce inflammation and that the development of inflammation in response to hypoxia may be clinically relevant. [11]. Previous studies have also demonstrated that total nitrate and nitrite $(\mathrm{NOx})$ production is lower in OSAS patients than in controls $[12,13]$. Both oxidative stress and inflammation are major components in the initiation and development of endothelial dysfunction, which is widely accepted as an early marker of atherosclerosis. Flow-mediated, brachial artery vasodilation (FMD) is a well established marker of endothelial function, as the result of endothelial release of NO [14-16]. Endothelial function, which is the result of a reduction in NO bioavailability, is markedly reduced in patients with moderate/severe OSAS [17-23]. Chronic low-grade inflammation, oxidative stress, metabolic abnormalities and endothelial dysfunction in OSAS could accelerate atherogenesis. However, so far, the biological mechanisms which may explain the association of OSA with endothelial dysfunction are still under debate.

Nasal continuous positive airway pressure (nCPAP) is first-line therapy for OSAS to reduce daytime sleepiness and improve cardiovascular and metabolic outcomes [24]. Previous studies have demonstrated a beneficial effect of nCPAP on several markers of oxidative stress in patients with OSAS [25-29]. An improvement of arterial dysfunction after nCPAP therapy has been also demonstrated [12,30-33].

Aim of our study was to assess the association between OSAS, endothelial dysfunction and oxidative stress in a sample of patients with different severity of OSAS. Further aim was to evaluate the effect of nCPAP on FMD and the levels of markers of systemic oxidative stress in patients with severe OSAS after using nCPAP for six months.

\section{Methods \\ Patients}

The study group consisted of 138 consecutive patients who were referred to our metabolic outpatients clinic because of suspected metabolic disorders with heavy snoring and possible OSAS. All patients had a complete clinical and biochemical work up, including polysomnography (PSG), as part of routine clinical examination.

Written consent was obtained from all subjects before the study and the study conforms to the ethical guidelines of the 1975 Declaration of Helsinki. The research protocol was approved by the University Department of Experimental Medicine and Pathology scientific board in 2004. According to the Hospital Ethical Committee guidelines, ethical approval was not required since the study was observational and did not include the use of new drugs and/or new experimental treatments. To be eligible for the study, patients had to fulfil the following criteria: no history and clinical signs of heart failure, autoimmune disease, acute inflammatory disease, or any severe disease shortening life expectancy, such as diagnosed cancer, chronic liver disease, severe renal disease.

Arterial blood pressure was measured on the right arm with the subjects in a sitting position and after a 5-min rest, using a mercury sphygmomanometer: the average of two measurements, 1 min apart, was considered. Waist circumference, height and weight were recorded with subjects wearing light clothing, without shoes and body mass index (BMI) was calculated as weight $(\mathrm{Kg})$ divided by height $\left(\mathrm{m}^{2}\right)$. MS was diagnosed following the International Diabetes Federation criteria [34]. Diabetes was diagnosed according to the WHO criteria. Subjects taking insulin or oral antidiabetic drugs were considered to have diabetes.

\section{Flow-mediated vasodilatation}

Ultrasound assessment of endothelial dependent and independent FMD of brachial artery was investigated according to the recently reported guideline [16]. Briefly, the study was performed in a temperature-controlled room $\left(22^{\circ} \mathrm{C}\right)$ with the subjects in a resting, supine state between 8 a.m. and 10 a.m. after at least a 8-hour fasting; brachial artery diameter was imaged using a 7.5-Mhz linear array transducer ultrasound system (Siemens) equipped with electronic callipers, vascular software for two-dimensional imaging, colour and spectral Doppler, and internal electrocardiogram; the brachial artery was imaged at a location 3-7 cm above the antecubital crease; to create a flow stimulus in the brachial artery, a sphygmomanometric cuff was placed on the forearm; the cuff was inflated at least $50 \mathrm{mmHg}$ above systolic pressure to occlude artery inflow for $5 \mathrm{~min}$; all vasodilatation measurements were made at the end of diastole; FMD was expressed as a change in post-stimulus diameter evaluated as a percentage of the baseline diameter.

\section{Blood sampling protocol}

Fasting venous blood samples were taken in the supine position on the morning after performing polysomnography and stored at $-80^{\circ} \mathrm{C}$ until assay. A final morning fasting blood sample was obtained after using CPAP treatment for a period of six months.

Subjects underwent routine biochemical evaluation including fasting total and HDL-cholesterol, triglycerides, glucose and insulin. Serum total cholesterol, HDLcholesterol and triglycerides were measured by an Olympus AN 560 apparatus using an enzymatic colorimetric method. LDL-cholesterol levels were calculated according to the Friedwald formula. Plasma insulin levels were assayed by commercially available radioimmunoassay. The homeostasis model assessment (HOMA-IR) was used to estimate insulin resistance using the formula: glucose $(\mathrm{mmol} / \mathrm{L}) \times$ [insulin $(\mathrm{mU} / \mathrm{L}) / 22$. 
A colorimetric assay kit (Tema Ricerca, Italy) was used to determine nitric oxide metabolites nitrite and nitrate (NOx) in the serum. Intra-assay and interassay coefficients of variation were $2.9 \%$ and $1.7 \%$ respectively.

Serum levels of soluble NOX2-derived peptide (sNOX2-dp) were detected by ELISA method as previously described [35]; intra-assay and inter-assay coefficients of variation were $5.2 \%$ and $6 \%$, respectively. Values are expressed as $\mathrm{pg} / \mathrm{ml}$.

Urinary 8-iso-prostaglandin F2 $\alpha$ (8-iso-PGF2 $\alpha$ ) was measured by a previously described and validated enzyme immunoassay method [36]. Intra-assay and inter-assay coefficients of variation were $2.1 \%$ and $4.5 \%$, respectively.

\section{Polysomnography (nocturnal recording)}

Patients underwent unattended overnight home PSG using a overnight home sleep recording (Embletta, PDS; Medcare, Reykjavik, Iceland). The device recorded nasal and oral airflow, chest and abdominal movements, and pulse oximetry. The sleep recordings were downloaded to a computer and scored by a principal investigator. A minimum of 5 hours of recording was accepted to be adequate for scoring. The presence and severity of apnea was assessed based on the number of apnoea/hypopnoea episodes per hour of sleep (apnoea/hypopnoea index AHI). Apnea was defined as continuous cessation of airflow for more than 10-s and hypopnoea was defined as reduction of airflow for more than-10 $\mathrm{s}$ with oxygen desaturation of $\geq 4 \%$ and arousal. OSAS was defined as an AHI of $\geq 5$. Patients were categorized into four subgroups according to OSA severity, as follows; normal AHI $<5$ events/h; mild OSA, AHI $<5$ to $<15$ events/h; moderate OSA, AHI $<15$ to $<30$ events/h; severe OSA, AHI $\geq 30$ events/h.

Patients had overnight home pulse oximetry monitoring with a transcutaneous fingertip sensor connected via cable to an OhmedaBiox 3700 pulse oximeter (Louisville, $\mathrm{CO})$. The mean haemoglobin oxygen saturation level $\left(\mathrm{SaO}_{2}\right)$ in total sleep time was also calculated.

Patients with severe OSAS underwent a full-night nCPAP titration study at home using an automated pressure setting device. The first consecutive 10 patients with severe OSAS who adhered to nCPAP treatment over a period of six months were revaluated.

Adherence to nCPAP was defined as nCPAP use for at least 4 hours per night and 5 days per week.

\section{Statistical analysis}

Statistical analysis was performed by using the SPSS statistical software version 11.0 for Windows (SPSS,Inc.,Chicago.Illinois). All variables were tested for normal distribution prior to analyses. Data are expressed as the mean \pm SD for continuous variables. The correlation between variables was analysed with the Pearson and the
Spearman tests. Student's t-test for unpaired data was used for the comparison of mean values. Group comparisons were performed by use of analysis of variance and test for linear trend in One-way ANOVA. Proportions and categorical variables were tested by the $x^{2}$-test and by the 2-tailed Fisher's exact method when appropriate. Multiple linear regression analysis was performed to determine the independent predictors of FMD. All $P$-values are two-tailed; a $P$-value of less than 0.05 was considered statistically significant.

\section{Results}

Out of the 138 patients who performed overnight polysomnography, 47 had a primary snoring and 91 had a positive polysomnography for OSA: 61 had mild/ moderate and 30 severe OSA.

Clinical and metabolic characteristics of patients with OSA by severity and control snorers are reported in Table 1. A strong positive association was observed between OSA severity and the indices of central obesity, i.e. body mass index $(\mathrm{p}=<0.01)$ and waist and hip circumferences $(\mathrm{p}<0.001)$. In addition, a positive association was observed with serum insulin and HOMA-IR level and with urinary 8-iso-PGF2 $\alpha$ concentration. As compared to non-OSAS, patients with severe OSAS had statistically significant higher urinary 8 -iso-PGF2 $\alpha$ and higher serum sNOX2-dp and lower NOx, although not at a statistically significant extent. Furthermore, a statistically significant negative association was observed between FMD and OSA severity.

Table 2 shows the prevalence of MS and its components in patients with different severity of OSAS. A statistically significant increase in the prevalence of central obesity was observed from snorers to subjects with severe OSAS $(\mathrm{p}=0.012)$, although a positive trend was also observed for the other components of MS. Fifty-six percent of patients with OSAS had MS, and 62.6\%, 30.0\% and $67.1 \%$ had hypertension, hyperlipidemia and hyperglycemia and/or type 2 diabetes, respectively.

Table 3 shows bivariate correlations between AHI and some clinical and metabolic characteristics. AHI was significantly correlated with the indices of central obesity and with urinary 8 -isoprostanes $(\mathrm{r}=0.298, \mathrm{p}<0.001)$. In turn, urinary 8-iso-PGF2 $\alpha$ were positively correlated with sNOX2-dp $(r=0.250, p<0.01)$ and negatively correlated with $\mathrm{NOx}(\mathrm{r}=0.360, \mathrm{p}<0.001)$. A negative correlation was also observed between FMD and waist circumference $(\mathrm{r}=-0.199 ; \mathrm{p}<0.05)$, serum insulin and triglyceride levels $(\mathrm{r}=-0.241 ; \mathrm{p}=0.01$ and $\mathrm{r}=-0.238$; $\mathrm{p}<0.01$ respectively) and the metabolic score $(\mathrm{r}=-0.335$; $\mathrm{p}<0.001)$.

In a stepwise multiple regression analysis including age, gender, waist circumference, $\mathrm{AHI}, \mathrm{SaO}_{2}, \mathrm{HOMA}-\mathrm{IR}$, total cholesterol, systolic blood pressure, diabetes, urinary 
Table 1 Clinical and metabolic characteristics of patients with OSA by severity and control snorers

\begin{tabular}{|c|c|c|c|c|}
\hline & \multicolumn{3}{|l|}{ OS A } & \multirow[b]{3}{*}{$\mathbf{P}$} \\
\hline & snorers & $\mathrm{mild} /$ moderate & severe & \\
\hline & $\overline{\mathrm{AHI}<5 \mathrm{n}=47}$ & $\overline{\text { AHI } 5-29 n=61}$ & $A H I \geq 30 n=30$ & \\
\hline Age & $51.3 \pm 11.7$ & $53.2 \pm 11.7$ & $56.6 \pm 9.8$ & NS \\
\hline Males (\%) & 66.0 & 70.5 & 83.3 & NS \\
\hline AHI (events/h) & $1.2 \pm 1.4$ & $14.6 \pm 8.0$ & $42.8 \pm 14.2$ & $<0.001$ \\
\hline Average $\mathrm{SaO}_{2}$ & $95,0 \pm 1,4$ & $93,6 \pm 2,2$ & $92,6 \pm 2,5$ & $<0,01$ \\
\hline Body Mass Index $\left(\mathrm{kg} / \mathrm{m}^{2}\right)$ & $29,3 \pm 3,9$ & $30,5 \pm 4,5$ & $32,8 \pm 5,1$ & $<0,01$ \\
\hline Waist circumference $(\mathrm{cm})$ & $102,5 \pm 11,1$ & $105,8 \pm 12,1$ & $113,6 \pm 12,1$ & $<0,001$ \\
\hline Hip circumference $(\mathrm{cm})$ & $106,5 \pm 8,5$ & $109,9 \pm 9,1$ & $116,9 \pm 11,7$ & $<0,001$ \\
\hline Blood glucose (mg/dl) & $103.1 \pm 24.5$ & $107.5 \pm 41.5$ & $107.1 \pm 28.1$ & 0.428 \\
\hline Insulin $(\mu \mathrm{U} / \mathrm{mL})$ & $14.0 \pm 6.7$ & $19.2 \pm 19.3$ & $24.4 \pm 22.4$ & $<0.05$ \\
\hline HOMA-IR & $3.6 \pm 1.9$ & $5.8 \pm 6.8$ & $8.4 \pm 11.1$ & $<0.01$ \\
\hline Total cholesterol (mg/dl) & $210.4 \pm 41.4$ & $201.4 \pm 38.7$ & $207.9 \pm 39.9$ & NS \\
\hline LDL-cholesterol (mg/dl) & $137.7 \pm 35.2$ & $128.0 \pm 33.7$ & $128.8 \pm 33.3$ & NS \\
\hline HDL-cholesterol (mg/dl) & $46.8 \pm 11.5$ & $46.0 \pm 11.5$ & $45.8 \pm 9.9$ & NS \\
\hline Triglycerides (mg/dl) & $131.1 \pm 65.8$ & $136.1 \pm 80.7$ & $167.5 \pm 134.0$ & NS \\
\hline Urinary 8-iso-PGF2a (pg/mg creatinine) & $284.0 \pm 77,3$ & $289.5 \pm 77,0$ & $337.6 \pm 74.5$ & $<0.001$ \\
\hline sNOX2-dp (pg/ml) & $26.2 \pm 8,2$ & $26.3 \pm 7.5$ & $28,8 \pm 8.0$ & NS \\
\hline Serum NOx (uM/ml) & $27.1 \pm 14.6$ & $27.0 \pm 12,5$ & $23,6 \pm 16.0$ & NS \\
\hline FMD\% & $6.2 \pm 3.2$ & $7.2 \pm 4.6$ & $4.9 \pm 2.7$ & $<0.05$ \\
\hline
\end{tabular}

8 -iso-PGF $2 \alpha$ and MS, as independent variable, MS $(\beta=$ $-0.40, \mathrm{t}=-4.63, \mathrm{p}<0.001)$ and urinary 8 -iso-PGF2 $\alpha(\beta=$ $-0.17, \mathrm{t}=-2.02, \mathrm{p}<0.05)$ were the only independent predictors of FMD $\left(\mathrm{R}^{2}=0.18\right)$.

Changes before and after CPAP treatment observed in the first consecutive 10 patients with severe OSAS who were compliant to CPAP treatment over a period of six months. are shown in Table 4. During treatment, mean HAI of the patients was significantly decreased from 43.4 \pm 12.6 at baseline to $7.0 \pm 5.0(\mathrm{p}<0.001)$, while mean $\mathrm{SaO}_{2}$ increased from $91.8 \pm 3.7$ to $97.0 \pm 1.0(\mathrm{p}=0.001)$. A statistically significant decrease of mean serum sNOX2-dp $(\mathrm{p}<0.005)$ and of urinary 8-iso-PGF2 $\alpha(\mathrm{p}<0.01)$ was observed, while serum NOx showed only a minor statistically non significant increase. A statistically significant increase of FMD was also observed (from 3.6\% to 7.0\%). During the six months treatment, no significant change in body weight or cardiovascular risk factors was observed.

\section{Discussion}

In our study, patients with severe OSA syndrome presented increased systemic oxidative stress. To assess oxidative stress in vivo, we measured urinary 8-isoprostanes, which are reliable markers of lipoperoxidation and, for the first time, sNOX2-dp, a marker of NOX2 activation by blood cells, which is a ROS generating enzyme implicated in arterial function via oxidative-stress mediated $\mathrm{NO}$ inactivation $[3,37,38]$.

Table 2 Prevalence of MS and its components in patients with different severity of OSAS

\begin{tabular}{|c|c|c|c|c|}
\hline & \multicolumn{3}{|l|}{ OS A } & \multirow[b]{3}{*}{$\mathbf{P}$} \\
\hline & snorers & mild/moderate & severe & \\
\hline & $\overline{\mathrm{AHI}}<5 \mathrm{n}=47$ & AHI $5-29 n=61$ & $\mathrm{AHI} \geq 30 \mathrm{n}=\mathbf{3 0}$ & \\
\hline Hyperglycemia* & 46 & 41 & 56,7 & NS \\
\hline Hypertension* & 51,1 & 60,7 & 66,7 & NS \\
\hline Central obesity* & 78,7 & 75,4 & 96,7 & $<0.05$ \\
\hline Low HDL-cholesterol* & 27,7 & 29,5 & 40 & NS \\
\hline Hypertriglyceridemia* & 21,3 & 23 & 40 & NS \\
\hline All Metabolic syndrome* & 46,8 & 49,2 & 70 & NS \\
\hline Metabolic syndrome* with diabetes & 8,5 & 23 & 16,7 & NS \\
\hline SCORE Metabolic Syndrome & $2.1 \pm 1.2$ & $2.3 \pm 1.4$ & $2.9 \pm 1.2$ & $<0.05$ \\
\hline
\end{tabular}

*according to IFD criteria (Ref. [34]). 
Table 3 Correlations between $\mathrm{AHI}$ and some clinical and metabolic characteristics

\begin{tabular}{lcc}
\hline & \multicolumn{2}{c}{ A H I } \\
\cline { 2 - 3 } & $\mathbf{r}$ & $\mathbf{P}$ \\
\hline Age & 0.132 & $\mathrm{NS}$ \\
\hline Average $\mathrm{SaO}_{2}$ & -0.386 & $<0,01$ \\
\hline Body Mass Index $\left(\mathrm{kg} / \mathrm{m}^{2}\right)$ & 0.271 & $<0,01$ \\
\hline Waist circumference $(\mathrm{cm})$ & 0.294 & $<0.001$ \\
\hline Hip circumference $(\mathrm{cm})$ & 0.295 & $<0.001$ \\
\hline Blood glucose $(\mathrm{mg} / \mathrm{dl})$ & 0.015 & $\mathrm{NS}$ \\
\hline Insulin $(\mu \mathrm{U} / \mathrm{mL})$ & 0.249 & $<0.01$ \\
\hline HOMA-IR & 0.284 & $<0.01$ \\
\hline Total cholesterol $(\mathrm{mg} / \mathrm{dl})$ & -0.034 & $\mathrm{NS}$ \\
\hline LDL-cholesterol $(\mathrm{mg} / \mathrm{dl})$ & -0.102 & $\mathrm{NS}$ \\
\hline HDL-cholesterol $(\mathrm{mg} / \mathrm{dl})$ & -0.055 & $\mathrm{NS}$ \\
\hline Triglycerides $(\mathrm{mg} / \mathrm{dl})$ & 0.056 & $\mathrm{NS}$ \\
\hline Urinary 8-iso-PGF2a $(\mathrm{pg} / \mathrm{mg}$ creatinine) & 0.298 & $<0.001$ \\
\hline sNOX2-dp $(\mathrm{pg} / \mathrm{ml})$ & 0.013 & $\mathrm{NS}$ \\
\hline Serum NOx $(\mathrm{uM} / \mathrm{ml})$ & -0.135 & $\mathrm{NS}$ \\
\hline FMD\% & -0.166 & $\mathrm{NS}$ \\
\hline Metabolic score & 0.159 & $\mathrm{NS}$ \\
\hline
\end{tabular}

In fact, we found that patients with OSAS had significantly higher 8 -iso-PGF2 $\alpha$ urinary levels than healthy controls and that the severity of OSA was significantly correlated with the oxidative stress. In addition, severe OSAS had a tendency towards higher serum sNOX2-dp and lower serum nitrate and nitrite (NOx), which are stable NO derivatives, reflecting overall NO production. In keeping with the above results, we found a statistically significant decreased FMD in patients with severe OSAS. At multivariate analysis, MS and urinary 8-iso-PGF $2 \alpha$ were independent predictors of FMD suggesting that in patients with OSA oxidative stress promotes arterial dysfunction likely via $\mathrm{NO}$ biosynthesis and/or inactivation. Moreover, as already reported, we further confirmed the strong association between MS and endothelial dysfunction [4].

Table 4 Changes before and after nCPAP treatment in 10 patients with severe OSAS

\begin{tabular}{lccc}
\hline & Baseline & After C-PAP & P \\
\hline Body Mass Index $\left(\mathrm{kg} / \mathrm{m}^{2}\right)$ & $35.3 \pm 5.4$ & $36.0 \pm 6.0$ & $\mathrm{NS}$ \\
\hline AHI (events/h) & $43.4 \pm 12.6$ & $7 \pm 5$ & 0.000 \\
\hline ODI (events/h) & $35.8 \pm 23.5$ & $2.8 \pm 1.6$ & 0.002 \\
\hline Average SaO $(\%)$ & $91.8 \pm 3.7$ & $97 \pm 1$ & 0.001 \\
\hline $\begin{array}{l}\text { Urinary 8-iso-PGF2a } \\
\text { (pg/mg creatinine) }\end{array}$ & $350.5 \pm 43.3$ & $269.6 \pm 48.4$ & 0.007 \\
\hline sNOX2-dp $(\mathrm{pg} / \mathrm{ml})$ & $38.2 \pm 7.4$ & $29 \pm 3.2$ & 0.003 \\
\hline Serum NOx $(\mathrm{uM} / \mathrm{ml})$ & $23.9 \pm 3.23$ & $27.3 \pm 5$ & $\mathrm{NS}$ \\
\hline FMD $(\%)$ & $3.64 \pm 4.2$ & $7.0 \pm 4.7$ & 0.015 \\
\hline
\end{tabular}

However, earlier studies of oxidative stress in OSAS provided conflicting results. Yamauchi et al. [39] found increased urinary 8-hydroxy-20-deoxyguanosine (8-OhdG) excretion in the severe OSA patients, Carpagnano et al. [10] observed elevated 8-isoprostane levels in the exhaled breath condensate in OSA patients and Dyugovskaya et al. [40] detected an increase in the production of ROS in OSA. Several other studies reported abnormal lipid peroxidation in OSAS $[7,8]$ or reduced total antioxidant status [9]. Conversely, Wali et al. [41] found no differences in susceptibility of LDL to oxidative stress and Svatikova et al. [42] reported that patients with moderate - severe OSA did not have evidence for greater oxidative stress and lipid peroxidation than healthy normal subjects. Negative results were also obtained in a recent study by Lee et al. [6] where no significant difference in either oxidative stress or antioxidant status markers was observed among normal patients and those with moderate and severe OSA; in this study, oxidative stress was related to central obesity rather than intermittent hypoxia and waist-to- hip ratio was a significant independent variable of oxidized-LDL, glutathione peroxidase, total antioxidant status and superoxide-dismutase.

In our study patients with OSA had reduced FMD, a widely used non invasive method to measure endothelial dysfunction. This is in agreement with the results reported by Oflaz et al. [19] and Grebe et al [43] who found a significant reduction of FMD in patients with OSA without other co-morbidities compared to healthy subjects; moreover, in the last study, the administration of i.v. vitamin $C$ improved endothelial function in OSAS patients, leading to an increase of FMD to a level comparable to that observed in the control group. Similar findings were obtained by Büchner et al. [26] who found that endothelial function in OSAS patients improved either after infusion of vitamin $\mathrm{C}$ or after nCPAP treatment.

Conversely, Kato et al. [44] found no differences in FMD between OSA patients free of other diseases and healthy controls, although endothelium-dependent vasodilation tested by use of forearm blood flow responses to intra-arterial infusion of acetylcholine showed a blunted vasodilation in OSA patients.

In studies conducted by Chung et al. [45], oxygen desaturation index was the only significant determinant of FMD. Finally, in keeping with the above studies, Ip et al. [46] demonstrated that subjects with OSA had lower FMD compared with subjects without OSA and that major determinants of FMD in OSA patients were AHI and age.

The above data raise the question whether OSA itself results in oxidative stress and arterial dysfunction or it is simply a consequence of metabolic comorbidities frequently associated to OSAS. In fact, in a previous paper, we provided evidence that MS patients have lower FMD and NOx serum levels and increased urinary 8- 
iso-PGF $2 \alpha$ and serum sNOX2-dp values, as compared to controls [47]. Thus, we acknowledge that MS and several potential confounders may influence oxidative stress in OSAS.

In our study, the first 10 patients with severe OSAS who adhered to nCPAP therapy were revaluated. Longterm compliance to nCPAP therapy was found to be effective in reducing the levels of systemic oxidative stress. In fact, nCPAP therapy normalized urinary 8 -iso-PGF2 $\alpha$ and serum sNOX2-dp values even though there was no significant change in body weight or cardiovascular risk factors during the six months treatment.

Our findings are in keeping with the results of several uncontrolled studies showing that short and long term nCPAP reduces oxidative stress in OSAS patients [7-10, $25,27,28]$.

Conversely, in a study of 41 moderate-severe OSAS without other diseases, $4 \mathrm{~h}$ treatment with nCPAP did not affect plasma levels of isoprostanes and other markers of oxidative stress [41]. Moreover, one night nCPAP therapy did not significantly change the susceptibility of LDL to peroxidation and the levels of the antioxidant enzymes [42].

In our study, FMD showed a statistically significant increase after nCPAP over a six months period. This is in keeping with recently reported data $[23,31,32,47]$. Moreover, FMD significantly improved 1 week and 4 weeks after nCPAP in 10 male OSA patients concomitantly with an increase of plasma NOx concentrations, and a correlation between the two was observed [12]. Finally, FMD and endothelial nitric oxide production increased whereas expression of NFkB and nitrotyrosine decreased in patients who adhered to CPAP $>4$ hours daily [48].

In addition, in keeping with the above results, an improvement of carotid intima thickness, an early sign of atherosclerosis, was also observed following four-months nCPAP therapy [33].

Strength of our study are, first, that in contrast to many previous studies performed in otherwise healthy OSAS, we included patients with concomitant chronic diseases such as obesity, metabolic syndrome, hypertension, type 2 diabetes, i.e. a representative sample of the real-world OSA population. Indeed, prevalence of MS in our severe OSAS was $70 \%$ and $67 \%$ had hypertension and none of these clinical features did change after nCPAP therapy. By contrast, most published papers refer to otherwise healthy OSAS, without cardiovascular comorbidity, poorly representative of the habitual overall OSA population. Therefore, this is the first study to demonstrate the efficacy of nCPAP therapy on oxidative stress and arterial dysfunction in OSA patients with cardiometabolic risk factors, independent from weight loss and risk factor management.

Secondly, we assessed oxidative stress by measuring urinary 8-isoprostanes, which are elevated in several metabolic and cardiovascular diseases and possibly involved in atherosclerosis development and progression. Thirdly, we measured for the first time sNOX2-dp, a marker of NOX2 activation, a member of the NADPH oxidase family which plays an important role in ROS production and the key mechanisms underlying the development of endothelial dysfunction and cardiovascular pathophisiology. Indeed, so far, a role of NADPH oxidase in oxidative and proinflammatory responses after hypoxia/reoxigenation patterns simulating severe sleep apnea oxygenation has been demonstrated in a murine model of sleep apnea [49].

Unattended home PSG should be considered as a major limitation of this study, although an excellent correlation between the results of attended PSG and home monitoring has been demonstrated [50]. Indeed, PSG and home sleep tests use the same respiratory equipment, pulse oximetry equipment, and movement and position sensors and data generated from each test is analyzed in the same manner. Home monitoring has also the ability to record in a natural sleep environment and patients are tested in the comfort and privacy of their home. Further limitation of the study is the rather small number of patients who were included in the nCPAP study. Finally, the low correlation coefficients between AHI index and markers of oxidative stress suggest caution in the interpretation of the results.

\section{Conclusions}

In conclusion, the results of our study indicate that patients with OSAS have an increased oxidative stress and arterial dysfunction that are partially reversed by nCPAP treatment.

We believe that our findings, which support a strong interplay between OSAS, MS, oxidative stress and arterial dysfunction, may be relevant for a better understanding of the pathogenesis of cardiovascular disorders in patients with OSAS.

\section{Competing interests}

The authors declare that they have no competing interests.

\section{Acknowledgments}

We wish to thank nurses Monica Brancorsini and Daniela Salzano for their skilful cooperation.

\section{Author details}

'Department of Internal Medicine and Medical Specialities, Sapienza University, Rome, Italy. ${ }^{2}$ Department of Public Health and Infectious Diseases, Division of Internal Medicine C, Policlinico Umberto 1, Viale del Policlinico 155, 00161 Rome, Italy.

\section{Authors' contribution}

MDB participated in the design and coordination of the study; MF and MB performed PSG studies and participated in the design of the study; LL and LP performed FMD studies; FA performed clinical studies; TA contributed to clinical data collection and elaboration: RC carried out the immunoassays; FV reviewed and edited data; FA wrote manuscript/study design and performed the statistical analysis; All authors read and approved the final manuscript. 


\section{Financial disclosure}

All the Authors have no relevant financial interest in this manuscript.

Received: 18 August 2011 Accepted: 23 July 2012

Published: 23 July 2012

\section{References}

1. Remmers JE, deGroot WJ, Sauerland EK, Anch AM: Pathogenesis of upper airway occlusion during sleep. J Appl Physiol 1978, 44:931-938.

2. Shamsuzzaman AS, Gersh BJ, Somers VK: Obstructive sleep apnoea: implications for cardiac and vascular disease. JAMA 2003, 290:1906-1914.

3. Lattimore JD, Celermajer DS, Wilcox I: Obstructive sleep apnea and cardiovascular disease. J Am Coll Cardiol 2003, 41:1429-1437.

4. Angelico F, Del Ben M, Augelletti T, et al: Obstructive sleep apnoea syndrome and the metabolic syndrome in an internal medicine setting. Eur J Intern Med 2010, 21:191-195.

5. Suzuki YJ, Jain V, Park AM, Day RM: Oxidative stress and oxidant signaling in obstructive sleep apnea and associated cardiovascular diseases. Free Radic Biol Med 2006, 40:1683-1692.

6. Lee SD, Ju G, Choi JA, Kim JW, Yoon IY: The association of oxidative stress with central obesity in obstructive sleep apnea. Sleep Breath 2011, 16:511-517.

7. Barceló A, Miralles C, Barbé F, Vila M, Pons S, Agustí AG: Abnormal lipid peroxidation in patients with sleep apnoea. Eur Respir J 2000, 16:644-647.

8. Lavie L, Vishnevsky A, Lavie P: Evidence for lipid peroxidation in obstructive sleep apnea. Sleep 2004, 27:123-128.

9. Katsoulis K, Kontakiotis T, Spanogiannis D: Total antioxidant status in patients with obstructive sleep apnea without comorbidities: the role of the severity of the disease. Sleep Breath 2011, 15:861-866

10. Carpagnano GE, Kharitonov SA, Resta O, Foschino-Barbaro MP, Gramiccioni E, Barnes PJ: 8-Isoprostane, a marker of oxidative stress, is increased in exhaled breath condensate of patients with obstructive sleep apnea after night and is reduced by continuous positive airway pressure therapy. Chest 2003, 124:1386-1392.

11. Eltzschig HK, Carmeliet P: Hypoxia and inflammation. N Engl J Med 2011, 364:656-665.

12. Ohike $Y$, Kozaki $K$, lijima $K$, et al: Amelioration of vascular endothelial dysfunction in obstructive sleep apnea syndrome by nasal continuous positive airway pressure-possible involvement of nitric oxide and asymmetric NG, NG-dimethylarginine. Circ J 2005, 69:221-226.

13. Ip MS, Lam B, Chan LY, et al: Circulating nitric oxide is suppressed in obstructive sleep apnea and is reversed by nasal continuous positive airway pressure. Am J Respir Crit Care Med 2000, 162:2166-2171.

14. Joannides $R$, Haefeli WE, Linder L: Nitric oxide is responsible for flow-dependent dilatation of human peripheral conduit arteries in vivo. Circulation 1995, 91:1314-1319.

15. Cooke JP: Flow, NO and atherogenesis. Proc Natl Acad Sci U S A 2003, 100:768-770.

16. Corretti MC, Anderson TJ, Benjamin EJ, et al: Guidelines for the ultrasound assessment of endothelial-dependent flow-mediated vasodilation of the brachial artery: a report of the International Brachial Artery Reactivity Task Force. J Am Coll Cardiol 2002, 39:257-265

17. Chung S, Yoon IY, Shin YK, et al: Endothelial dysfunction and C-reactive protein in relation with the severity of obstructive sleep apnea syndrome. Sleep 2007, 30:997-1001.

18. Nieto FJ, Herrington DM, Redline S, Benjamin EJ, Robbins JA: Sleep apnea and markers of vascular endothelial function in a large community sample of older adults. Am J Respir Crit Care Med 2004, 169:354-360.

19. Oflaz H, Cuhadaroglu C, Pamukcu B, et al: Endothelial function in patients with obstructive sleep apnea syndrome but without hypertension.Respiration 2006, 73:751-756.

20. Chung S, Yoon IY, Lee CH, Kim JW: The association of nocturnal hypoxemia with arterial stiffness and endothelial dysfunction in male patients with obstructive sleep apnea syndrome. Respiration 2010, 79:363-369.

21. Chung S: Endothelial dysfunction and inflammatory reactions of eldery and middle aged men with obstructive sleep apnea syndrome. Sleep Breath 2009, 13:11-17.
22. Duchna HW, Stoohs R, Guilleminault C, et al: Vascular endothelial dysfunction in patients with mild obstructive sleep apnea syndrome. Wien Med Wochenschr 2006, 156:596-604.

23. Jurado-Gámez B, Fernandez-Marin MC, Gómez-Chaparro JL, et al: Relationship of oxidative stress and endothelial dysfunction in sleep apnoea. Eur Respir J 2011, 37:873-879.

24. Fleetham J, Ayas N, Bradley D, CTS Sleep Disordered Breathing Committee, et al: Canadian Thoracic Society guidelines: diagnosis and treatment of sleep disordered breathing in adults. Can Respir J 2006 13:387-392.

25. Christou K, Kostikas K, Pastaka C, Tanou K, Antoniadou I, Gourgoulianis Kl: Nasal continuous positive airway pressure treatment reduces systemic oxidative stress in patients with severe obstructive sleep apnea syndrome. Sleep Med 2009, 10:87-94.

26. Büchner NJ, Quack I, Woznowski M, Stähle C, Wenzel U, Rump LC: Microvascular endothelial dysfunction in obstructive sleep apnea is caused by oxidative stress and improved by continuous positive airway pressure therapy.Respiration 2011, 82:409-417

27. Murri M, Alcázar-Ramírez J, Garrido-Sánchez L, et al: Oxidative stress and metabolic changes after continuous positive airway pressure treatment according to previous metabolic disorders in sleep apnea-hypopnea syndrome patients. Trans/ Res 2009, 3:111-121.

28. Hernandez C, Abreu J, Abreu P, Colino R, Jimenez: Effects of nasal positive airway pressure treatment on oxidative stress in patients with sleep apnea-hypopnea sindrome. Arch Bronconeumol 2006, 42:125-126.

29. Jelic S, Padeletti M, Kawut SM, et al: Inflammation, oxidative stress, and repair capacity of the vascular endothelium in obstructive sleep apnea. Circulation 2008, 117:2270-2278.

30. Comondore VR, Cheema R, Fox J, et al: The impact of CPAP on cardiovascular biomarkers in minimally symptomatic patients with obstructive sleep apnea: a pilot feasibility randomized crossover trial. Lung 2009, 187:17-22.

31. Bayram NA, Ciftci B, Keles T, et al: Endothelial function in normotensive men with obstructive sleep apnea before and 6 months after CPAP treatment. Sleep 2009, 32:1257-1263.

32. Ciccone MM, Favale $S$, Scicchitano $P$, et al: Reversibility of the endothelial dysfunction after CPAP therapy in OSAS patients. Int J Cardiol 2011, 158:383-386.

33. Drager LF, Bortolotto LA, Figueiredo AC, Krieger EM, Lorenzi GF: Effects of continuous positive airway pressure on early signs of atherosclerosis in obstructive sleep apnea. Am J Respir Crit Care Med 2007, 176:706-712.

34. Alberti KG, Zimmet P, Shaw J: Metabolic syndrome-a new world-wide definition. A Consensus Statement from the International Diabetes Federation. Diabet Med 2006, 23:469-480.

35. Pignatelli $P$, Carnevale R, Cangemi R, et al: Atorvastatin inhibits gp91phox circulating levels in patients with hypercholesterolemia. Arterioscler Thromb Vasc Biol 2010, 30:360-367.

36. Wang Z, Ciabattoni G, Créminon C, et al: Immunological characterization of urinary 8-epi-prostaglandin F2 alpha excretion in man. J Pharmacol Exp Ther 1995, 275:94-100.

37. Praticò D: Prostanoid and isoprostanoid pathways in atherogenesis. Atherosclerosis 2008, 201:8-16.

38. Cave AC, Brewer AC, Narayanapanicker A, et al: NADPH oxidases in cardiovascular health and disease. Antioxid Redox Signal 2006, 8:691-728.

39. Yamauchi $M$, Nakano $H$, Maekawa J, et al: Oxidative stress in obstructive sleep apnea. Chest 2005, 127:1674-1679.

40. Dyugovskaya L, Lavie P, Lavie L: Increased adhesion molecules expression and production of reactive oxygen species in leukocytes of sleep apnea patients.Am J RespirCrit Care Med 2002, 165:934-939.

41. Wali SO, Bahammam AS, Massaeli H, et al: Susceptibility of LDL to oxidative stress in obstructive sleep apnea.Sleep 1998, 21:290-296.

42. Svatikova A, Wolk R, Lerman LO, et al: Oxidative stress in obstructive sleep apnoea. Eur Heart J 2005, 26:2435-2439.

43. Grebe M, Eisele HJ, Weissmann N, et al: Antioxidant vitamin C improves endothelial function in obstructive sleep apnea. Am J RespirCrit Care Med 2006, 173:897-901

44. Kato M, Roberts-Thomson P, Phillips BG, et al: Impairment of endotheliumdependent vasodilation of resistance vessels in patients with obstructive sleep apnea. Circulation 2000, 102:2607-2610. 
45. Chung S, Yoon IY, Lee CH, Kim JW: The effects of nasal continuous positive airway pressure on vascular functions and serum cardiovascular risk factors in obstructive sleep apnea syndrome. Sleep Breath 2011, 15:71-76.

46. Ip MS, Tse HF, Lam B, Tsang KW, Lam WK: Endothelial function in obstructive sleep apnea and response to treatment. Am J RespirCrit Care Med 2004, 169:348-353.

47. Angelico F, Loffredo L, Pignatelli $P$, et al: Weight loss is associated with improved endothelial dysfunction via NOX2-generated oxidative stress down-regulation in patients with the metabolic syndrome. Intern Emerg Med 2011, 7:219-227.

48. Jelic S, Lederer DJ, Adams T, et al: Vascular inflammation in obesity and sleep apnea. Circulation 2010, 121:1014-1021.

49. Zhan G, Serrano F, Fenik P, et al: NADPH oxidase mediates hypersomnolence and brain oxidative injury in a murine model of sleep apnea. Am J Respir Crit Care Med 2005, 172:921-929.

50. Dingli $\mathrm{K}$, Coleman $\mathrm{EL}$, Vennelle $\mathrm{M}$, et al: Evaluation of a portable device for diagnosing the sleep apnoea/hypopnoea syndrome. Eur Respir J 2003, 21:253-259.

doi:10.1186/1471-2466-12-36

Cite this article as: Del Ben et al: Oxidative stress mediated arterial dysfunction in patients with obstructive sleep apnoea and the effect of continuous positive airway pressure treatment. BMC Pulmonary Medicine 2012 12:36

\section{Submit your next manuscript to BioMed Central and take full advantage of:}

- Convenient online submission

- Thorough peer review

- No space constraints or color figure charges

- Immediate publication on acceptance

- Inclusion in PubMed, CAS, Scopus and Google Scholar

- Research which is freely available for redistribution 